\title{
Microaneurysms Detection Using a Novel Neighborhood Analysis
}

\author{
Ivo Soares $^{1,2}$, Miguel Castelo-Branco ${ }^{1}$, and António M.G. Pinheiro ${ }^{2}$ \\ ${ }^{1}$ CICS - Research Centre of Health Sciences, University of Beira Interior \\ ${ }^{2}$ Remote Sensing Unit, University of Beira Interior \\ Covilhã, Portugal
}

\begin{abstract}
The earliest sign of the diabetic retinopathy is the appearance of small red dots in retinal fundus images, designated by microaneurysms. In this paper a scale-space based method is proposed for the microaneurysms detection. Initially, the method performs a segmentation of the retinal vasculature and defines a global set of microaneurysms candidates, using both coarser and finer scales. Using the finer scales, a set of microaneurysms candidates are analysed in terms of shape and size. Then, a set of gaussian-shaped matched filters are used to reduce the number of false microaneurysms candidates. Each candidate is labeled as a true microaneurysm using a new neighborhood analysis method. The proposed algorithm was tested with the training Retinopathy Online Challenge (ROC) dataset, revealing a $47 \%$ Sensitivity with an average number of 37.9 false positives per image.
\end{abstract}

Keywords: Biomedical image processing, Microaneurysms detection, Scale-space, Segmentation

\section{Introduction}

Diabetic retinopathy (DR) is one of the major causes of visual loss in the developed world. This disease can be prevented from causing blindness if treated in early stages, where the detection of microaneurysms (MAs) has proven to be a key factor [1]. Furthermore, it was found that the appearance and disappearance of the MAs in the initial stages of the retinopathy, designated by MA turnover, allows retinal experts to control the evolution of the disease [2]. Hence, an automatic detection method for the MAs would provide an invaluable help in the diagnosis and progression control of diabetic retinopathy. This is currently done manually, being a laborious task and very susceptible to observer error and fatigue. Although the recent research that has been developed, automatic detection of MAs is far from being trivial. The variations in size, shape, the influence of other retinal structures and illumination variation between retinal images, makes the automatic MAs detection and recognition very challenging. Most of the proposed methods for the detection of MAs follows a sequence of operations: Image preprocessing, candidate extraction, and classification $[1,3,4]$. 


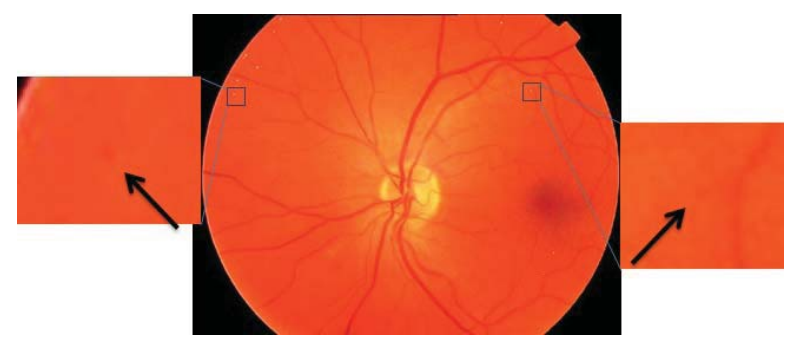

Fig. 1: Retinal image with microaneurysms labeled by the proposed method. The black arrows represent detected microaneurysms.

The preprocessing stage aims to reduce noise and improve contrast. The candidate extraction stage uses a segmentation technique to determine the maximum possible number of MAs candidates. Finally, the classification stage extracts some local features, used to classify MAs candidates as true or false using a supervised learning method. Examples of this state-of-the-art methods are by e.g. [5-8]. Other methods were proposed that do not require supervising classification like $[1,9]$. In this paper, a new method is proposed that do not require any supervised classification.

\section{Methods}

The proposed method comprises several steps. The first step is the construction and definition of the scale-space. Based on the defined scale-space, a segmentation of the vasculature is performed followed by the preliminary determination of MAs candidates. Next, a set of finer scales are used to reduce the microaneurysms candidates using a set of gaussian-shaped matched filters. Finally, each candidate is labeled as a true microaneurysm using a new neighborhood analysis. Since one of the main characteristics of MAs is its small size, a detailed evaluation of the influence of the scale is also presented.

\subsection{Scale-space definition}

The green channel component $I_{G}$ of each $R G B$ retinal image is selected, since it offers the best contrast and provides the most relevant clinical visual information [10]. No contrast normalization or enhancement is performed. Next, if $I_{G}$ has either a width or height smaller than 1000 pixels, it is proportionally resized using a bicubic interpolation, leading to a dimension of 1000 pixels in the smaller direction. Since MAs have a circular shape with a gaussian profile, they are ideal objects to be analysed using a scale-space approach [8]. Furthermore, as pointed in [11], a selective noise reduction process that retains the contrast of MAs is fundamental for an effective MA candidates extraction. Using the definitions of the linear scale-space image representation [12], a family of derived images is defined by the convolution of $I_{G}(\mathbf{x})$ with the Gaussian filter $g(\mathbf{x} ; t)$, given 
by $L(\mathbf{x} ; t)=g(\mathbf{x} ; t) * I_{G}(\mathbf{x}), \forall t$, where $g(\mathbf{x} ; t)=\frac{1}{\sqrt{2 \pi t}} e^{-\frac{|\mathbf{x}|^{2}}{2 t}} .2 t$ is the variance of the Gaussian filter, which defines the scale level. Larger $t$ values, result in stronger smoothing of $I_{G}(\mathbf{x})$, removing the details that are significantly smaller than $t[12]$. Since MAs are relatively small retinal structures, the Gaussian filter must provide a slow evolution and effective noise reduction while the features of interest are preserved. Hence, the gaussian kernel with a variance $\sigma^{2}=\sqrt{2} / 2$, was applied,

$$
W=\frac{1}{16}\left[\begin{array}{lll}
1 & 2 & 1 \\
2 & 4 & 2 \\
1 & 2 & 1
\end{array}\right]
$$

Since $\sigma^{2}=2 t$, the parameter $t$ can be defined at $N$ different scales according to $t_{n}=n \frac{\sqrt{2}}{4}$, with $n \in\{1, \ldots, N\}$. The Gaussian scale-space representation is defined as $L\left(\mathbf{x} ; t_{n}\right)$, with $n \in\{0, \ldots, N\} \quad(n=0$ represents the resized initial image). In this work $N$ was set to 10 . Considering the image $L\left(\mathbf{x} ; t_{n}\right)$ as an intensity surface, it is possible to describe the local shape characteristics of an image in a defined scale and coordinates using the Hessian matrix,

$$
H\left(\mathbf{x} ; t_{n}\right)=\left[\begin{array}{ll}
L_{x x}\left(\mathbf{x} ; t_{n}\right) & L_{x y}\left(\mathbf{x} ; t_{n}\right) \\
L_{y x}\left(\mathbf{x} ; t_{n}\right) & L_{y y}\left(\mathbf{x} ; t_{n}\right)
\end{array}\right] .
$$

$L_{x x}\left(\mathbf{x} ; t_{n}\right), L_{y y}\left(\mathbf{x} ; t_{n}\right)$ and $L_{x y}\left(\mathbf{x} ; t_{n}\right)$ are the second-order partial derivatives of $L\left(\mathbf{x} ; t_{n}\right)$ evaluated at the coordinates $\left(\mathbf{x} ; t_{n}\right)$. It is also possible to define the mean curvature at the scale $t_{n}$ as, $K\left(\mathbf{x} ; t_{n}\right)=\left(\lambda_{1}\left(\mathbf{x} ; t_{n}\right)+\lambda_{2}\left(\mathbf{x} ; t_{n}\right)\right) / 2$, where $\lambda_{1}\left(\mathbf{x} ; t_{n}\right)$ and $\lambda_{1}\left(\mathbf{x} ; t_{n}\right)$ are the minimum and maximum eigenvalues, respectively, of the Hessian matrix.

\subsection{Vasculature segmentation}

To perform the vasculature segmentation, $K\left(\mathbf{x} ; t_{n}\right)$ is normalized between 0 and 1 , followed by a histogram equalization (64 levels), denoted by $K_{h e}\left(\mathbf{x} ; t_{n}\right)$. This procedure enhances dark regions like vessels and MAs. For an effective vasculature segmentation both fine and thick vessels must be consider, i.e., both finer and broader scales must be analysed. Furthermore, at each scale only the regions in $K_{h e}\left(\mathbf{x} ; t_{n}\right)$ with high response are relevant. Hence, the vascular segmentation $V$ can be calculated by thresholding $K_{h e}\left(\mathbf{x} ; t_{n}\right)$ by $\alpha$ at each scale $t_{n}$, and summing the result across the previous described scale-space, i.e. $V=\sum_{n=1}^{N}\left(K_{h e_{\alpha}}\right)$ with $K_{h e_{\alpha}}=K_{h e}\left(\mathbf{x} ; t_{n}\right)$ when is larger or equal than $\alpha$ and 0 otherwise After testing, $\alpha$ was set to 0.95 . Then, $V$ is binarized by replacing all values above 0 with 1 , creating $V_{b}$. To improve the vascular segmentation, a two-level morphological closing is applied to $V_{b}$. The morphological closing operation is defined as $f \bullet b_{d}=\left(f \oplus b_{d}\right) \ominus b_{d}$ where $f$ is a generic $2 D$ function, $b_{d}$ is a disk-shaped structuring element with $d$ pixels size, and $\oplus$ and $\ominus$ are the morphological dilation and erosion, respectively. Initially a morphological closing with a structuring element $b_{1}$ is applied to $V_{b}$. Next, all the regions smaller than 200 pixels are removed. Finally, a second morphological closing with a structuring element $b_{5}$ is performed. This two-level closing operation favours the connection of vessels branches. 


\subsection{Initial MAs candidates determination}

The determinant of the second-order matrix at scale $t_{n}$, given by $\left|H\left(\mathbf{x} ; t_{n}\right)\right|=$ $\lambda_{1}\left(\mathbf{x} ; t_{n}\right) \lambda_{2}\left(\mathbf{x} ; t_{n}\right)$ has a high response to the MAs [13]. Hence, a simple way to calculate initial MAs regions is by binarizing $\left|H\left(\mathbf{x} ; t_{n}\right)\right|$ at each scale $t_{n}$ with an appropriate threshold $\beta$. Since the scale-space was constructed considering feature preservation across the scales, it was imposed as a condition that the MA candidate must be present at all binarized scales, i.e., $C=\sum_{n=1}^{N}\left|H\left(\mathbf{x} ; t_{n}\right)\right|_{\beta}$ with $\left|H\left(\mathbf{x} ; t_{n}\right)\right|_{\beta}=\left|H\left(\mathbf{x} ; t_{n}\right)\right|$ when is larger than $\beta$ and 0 otherwise. $C$ can be understood as the global MAs candidates localization, allowing the assessment of the existence of MAs in a particular region. The threshold $\beta$ is the parameter with highest influence in the final results, and a detailed study was made to assess its influence (Table 1). After extensive testing, $\beta$ was set to 225 .

\subsection{MAs finer scales assessment}

Typically the MAs analysis uses the single scale $\sigma=2[5,13]$. In order to evaluate the impact of different scales in the MAs detection, a set of finer scales was considered and analysed individually. The considered set of scales were $t_{k}=k \frac{\sqrt{2}}{4}$, with $k=2,3,4$ and 5 . Finer scales than the considered $t_{k}$ were considered too noisy, while coarser scales results in the lost of significant details. At the scale $t_{k}$ the MA candidates, defined by $C_{t_{k}}$ are extracted by thresholding $K_{h e}\left(\mathbf{x} ; t_{k}\right)>\alpha$. Since the MAs have a roughly circular shape and a small size, all the regions in $C_{t_{k}}$ larger than 100 pixels and with eccentricity [14] larger than 0.95 are removed. Next, the regions in $C_{t_{k}}$ that intersect the vascular segmentation $V_{b}$, or without any region with intersections of the global MA candidates $C$ are discarded. Finally a gaussian-shape matched filter is used to define the MA candidates. This filter is a template matching algorithm that is widely used in the detection of the blood vessels in retinal images [15]. The kernel can be defined by $m(\mathbf{x})=-\exp \left(-x^{2} / 2 s^{2}\right), \quad \forall|y| \leq L / 2$, where $L$ is the length of the vessel segment that has the same orientation and $s$ defines the spread of the vessel intensity profile [15]. For the vessels detection, the kernel is rotated at all possible vessel orientations and the maximum response from the filter bank is registered. Since MAs are different from the retinal vessels, a few modifications must be introduced. A blob detector at the scale $t_{k}$ can be defined as $B\left(\mathbf{x}, t_{k}\right)=\operatorname{Lx} x\left(\mathbf{x}, t_{k}\right)+\operatorname{Lyy}\left(\mathbf{x}, t_{k}\right)$, where $B\left(\mathbf{x}, t_{k}\right)$ has a larger response at dark structures as it is the case of MAs. The normalized cross-correlation $\hat{R}$, between a $2 D$ generic function $f(\mathbf{x})$ and the kernel $m(\mathbf{x})$ with a length $L$, standard-deviation $s$ and orientation $\theta$, can be defined as $\hat{R}(f(\mathbf{x}), m(\mathbf{x}, L, s, \theta))$. After testing, were considered the values $L=10$ and $s=\{1,1.25,1.50,1.75,2\}$, and $\theta$ was rotated by an amount of $10^{\circ}$. Based on this definitions, the operation given by $\rho=\max _{\forall \theta, s}\left\{\hat{R}\left(B\left(\mathbf{x}, t_{k}\right), m(\mathbf{x}, 10, s, \theta)\right)\right\}$ was defined. $\rho$ represents the normalized cross-correlation of $B\left(\mathbf{x}, t_{k}\right)$ with the filter $m(\mathbf{x})$. Theoretically the minimum value that $\rho$ can have in MAs regions is 0 . It was found after testing that the regions in $C_{t_{k}}$ with a minimum response of $\rho$ higher than 0.1 could be removed, leading to a larger reduction of the number of false regions. 


\subsection{MAs neighborhood analysis}

At this stage it is required to label the MA candidates as true or false. One of the main clues for the selection of MAs regarding the retinal background, is the difference in intensity values between the MA and its surroundings [11]. In this work a two stages labelling algorithm is presented that relies in the analysis of the MA and its surroundings. The first stage performs an analysis of the initial shape of the MA candidate (described in Fig. 2). Initially, a ridge [16] descriptor and its watershed are calculated. Based on the watershed regions, the neighborhood of the MA is defined. Next, it is determined if a reshape of the MA candidate is necessary. Following the algorithm of Fig. 2 some neighborhood regions might be merged with the initial MA candidate region. This merging is made at most on two different steps (controlled by the accumulator $j$ ). This leads to a more effective MA labeling. Furthermore, during the reshape process it is also possible to discard false MA candidates (see Fig. 2 for details). The second stage in the MA labelling, described in Fig. 3 defines if the remaining reshaped MA candidates are true or false candidates. In this last stage, the perimeter of each MA candidate is successively dilated and compared with the neighborhood regions defined by a watershed function of the ridge descriptor image. If in any of these dilations the median of the ridge values of the neighborhood is larger than the median of the ridge values of the dilated perimeter, the candidate is labeled as a true MA (see Fig. 3 for details).

\section{Results}

The publicly available Retinopathy Online Challenge dataset (ROC) [17] was used to analyse the performance of the proposed algorithm. The ROC dataset is composed of both a Training and a Test dataset, composed of 50 images each. Only the training dataset was used because is the only one with a groundtruth. Each scale in the previously defined set $t_{k}$ was considered and analysed individually, together with several values of $\beta$. Table 1 summarizes the results for this evaluation in terms of Sensitivity and mean value of False Positives per Image (FP/I). Fig. 4 shows the Receiver Operating Characteristic curves (ROCC) for each scale $t_{k}$. Table 1 also reveals that the variation of the scale $t_{k}$ and threshold $\beta$ do not have a strong influence in the recognition. These results were expected since the scale-space was specifically constructed for feature preservation. Moreover, can also be observed that the highest Sensitivity is obtained in the scale $t_{4}$. Hence from the analysed scales, $t_{4}$ is the best one to perform the MA detection. The best performance is achieved when $\beta=225$, resulting in a Sensitivity of $47 \%$ with $37.8 \mathrm{FP} / \mathrm{I}$. These results are confirmed in Fig. 4 where the scale $t_{4}$ have the larger area under the curve. Moreover, this result reveals to be competitive when compared with the results achieved in [11], as shown in Table 2. Other interesting results are highlighted with blue in Table 1. It is also important to observe that the scale $t_{5}$ also provides a good performance. 


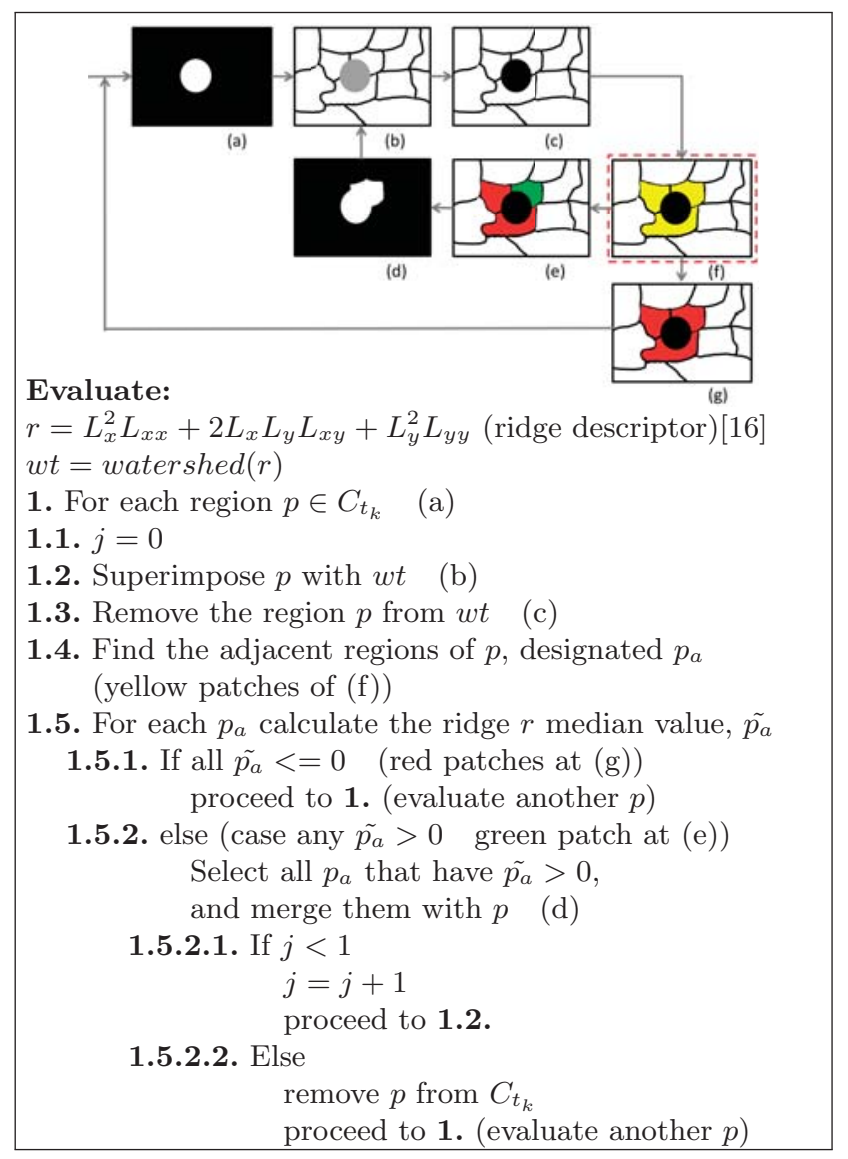

Fig. 2: MA candidate shape definition.

\section{Discussion}

In this paper, a very effective methodology for the automatic detection of MAs is presented. Furthermore, this paper provides several important contributions: 1) a space-scale specifically constructed and defined for the MAs extraction, 2) a new neighborhood analysis to label a MA candidate as a true or false MA, and 3) it performs a detailed analysis of the scale influence in the MAs detection. Although the method reveals a very good performance, new improvements might be considered in the future. As indicated in $[8,13]$ contrast normalization and shade correction can improve the selection of MA candidates. The scales $t_{4}$ and $t_{5}$ result in $\sigma=1.68$ and $\sigma=1.88$ respectively, which agrees with the typical $\sigma$ value defined in the bibliography $[5,18]$. An integration of both $t_{4}$ and $t_{5}$ scales will also be studied as future work, due to the good results achieved in both scales. 


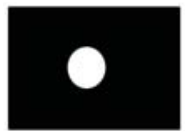

(a)

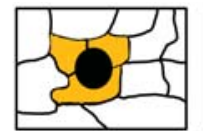

(b)

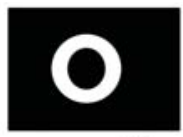

(c)

Evaluate:

Compute $r_{n}$ as the normalization of $r$ between 0 and 1 .

1. For each reshaped region $p \in C_{t_{k}}$

(a)

1.2. Find the yellow adjacent regions of $p$ (as explained in Fig. 2)

1.3. For all the yellow adjacent regions, calculate the $r_{n}$ median value, $\tilde{p}$

1.4. For $\epsilon=2$ to 7

1.4.1. Evaluate $d_{p}=\left(p \bigoplus b_{\epsilon}\right)-p \quad$ (c)

1.4.2. Calculate the $r_{n}$ median value of $d_{p}, \tilde{d}_{p}$

1.4.3. If $\tilde{p}>\tilde{d}_{p}$ label $p$ as MA proceed to $\mathbf{1}$.

1.4.4. Else if $\tilde{p}<=\tilde{d}_{p} \wedge \epsilon=7$ remove $p$ from $C_{t}$ proceed to 1 .

Fig. 3: MAs final labeling.

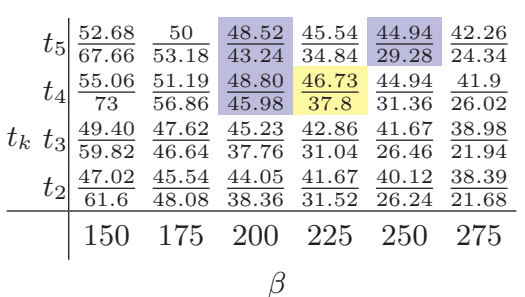

Table 1

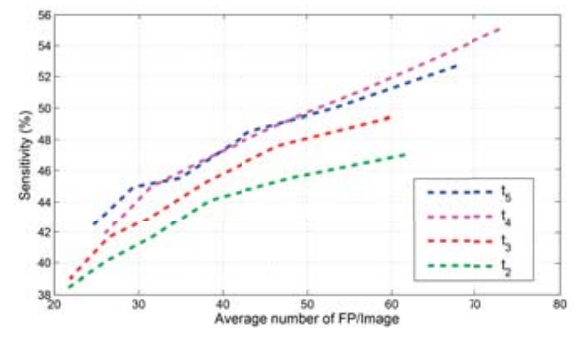

Fig. 4

Table 1: shows the relation between the scales $t_{k}$ and $\beta$ in the form $\frac{\text { Sensitivity }}{\text { FP/Image }}$ (considering a lesion-based criteria). The highlighted yellow value represents the best performance. Other interesting results are highlighted in blue. Figure 4 represents the ROCC for the scales set $t_{k}$.

\begin{tabular}{|l|c|c|}
\hline Algorithm & Sensitivity & FP/I \\
\hline Mizutani [11] & $45 \%$ & 27 \\
\hline Our method & $47 \%$ & 37.8 \\
\hline
\end{tabular}

Table 2: Comparison of the results using the ROC Training dataset.

\section{References}

1. I. Lazar and A. Hajdu, "Microaneurysm detection in retinal images using a rotating cross-section based model," in Biomedical Imaging: From Nano to Macro, 2011 
IEEE International Symposium on, 2011, pp. 1405-1409.

2. S. Nunes, I. Pires, A. Rosa et al., "Microaneurysm turnover is a biomarker for diabetic retinopathy progression to clinically significant macular edema: findings for type 2 diabetics with nonproliferative retinopathy," Ophthalmologica, vol. 223, no. 5, pp. 292-7, 2009.

3. K. Huang and M. Yan, "A local adaptive algorithm for microaneurysms detection in digital fundus images," in Computer Vision for Biomedical Image Applications, ser. Lecture Notes in Computer Science. Springer Berlin Heidelberg, 2005, vol. 3765 , pp. 103-113.

4. B. Antal and A. Hajdu, "Improving microaneurysm detection in color fundus images by using context-aware approaches." Comp. Med. Imag. and Graph., vol. 37, no. 5-6, pp. 403-408, 2013.

5. M. Niemeijer, J. Staal, M. Abramoff et al., "Automatic detection of red lesions in digital color fundus photographs," IEEE Transactions on Medical Imaging, vol. 24, p. $584592,2005$.

6. G. Quellec, M. Lamard, P. Josselin et al., "Optimal wavelet transform for the detection of microaneurysms in retina photographs," IEEE Trans. on Medical Imaging, vol. 27, p. 12301241, 2008.

7. B. Zhang, X. Wu, J. You et al., "Hierarchical detection of red lesions in retinal images by multiscale correlation," SPIE Medical Imaging, vol. 7260, p. 72601L, 2009.

8. B. Antal and A. Hajdu, "An ensemble-based system for microaneurysm detection and diabetic retinopathy grading," Biomedical Engineering, IEEE Transactions on, vol. 59, no. 6, pp. 1720-1726, June 2012.

9. L. Giancardo, F. Meriaudeau, T. Karnowski et al., "Microaneurysms detection with the radon cliff operator in retinal fundus images," SPIE Medical Imaging, vol. 7623 , p. 29,2010

10. R. J. Winder, P. J. Morrow, I. N. McRitchie et al., "Algorithms for digital image processing in diabetic retinopathy." Comp. Med. Imag. and Graph., vol. 33, no. 8, pp. 608-622, 2009

11. Y. Hatanaka et al., "Automated microaneurysm detection method based on doublering filter and feature analysis in retinal fundus images," in Computer-Based Medical Systems (CBMS), 2012 25th International Symposium on, June 2012, pp. 1-4.

12. A. Witkin, "Scale-space filtering," IJCAI'83, vol. 2, pp. 1019-1022, 1983.

13. K. Adal, S. Ali, D. Sidibé et al., "Automated detection of microaneurysms using robust blob descriptors," Proc. SPIE, vol. 8670, pp. $86700 \mathrm{~N}-86700 \mathrm{~N}-7,2013$.

14. M. U. Akram, S. Khalid, and S. A. Khan, "Identification and classification of microaneurysms for early detection of diabetic retinopathy," Pattern Recognition, vol. 46, no. 1 , pp. $107-116,2013$.

15. S. Chaudhuri, S. Chatterjee, N. Katz et al., "Detection of blood vessels in retinal images using two-dimensional matched filters," Medical Imaging, IEEE Transactions on, vol. 8, no. 3, pp. 263-269, 1989.

16. T. Lindeberg, "Scale-space theory: A basic tool for analysing structures at different scales," Journal of Applied Statistics, pp. 224-270, 1994.

17. M. Niemeijer, B. van Ginneken, M. Cree et al., "Retinopathy online challenge: Automatic detection of microaneurysms in digital color fundus photographs," Medical Imaging, IEEE Transactions on, vol. 29, no. 1, pp. 185-195, 2010.

18. B. Antal and A. Hajdu, "An ensemble-based microaneurysm detector for retinal images," in Image Processing (ICIP), 2011 18th IEEE International Conference on, Sept 2011, pp. 1621-1624. 